\title{
Survey on Web Image Re-Ranking Using Query- Specific Semantic Signatures
}

\author{
Vandana Ramchandra Nangare \\ Pune University, Department of Computer Engineering, Zeal College of Engineering \& Research, Narhe, Pune- 411041
}

\begin{abstract}
In today's world accessing of an image using search engine is a important task, but getting a relevant image is a complex task. To improve the result of web-based image search image re-ranking is effective way. In web based image search like google, bing use text query or query keyword to retrieve the images. From this pool of images user select one image as query image and remaining images re-ranked based on their visual similarities. A main contend is, the similarities of visual features do not well correlate with semantic meanings of images which understand users' search purpose. Commonly used social multimedia websites like Photo bucket, flicker, Amazon is used to retrieve images in such a large network is very useful but also very monotony or challenging process because there exists lots of information such as images, text and network structure. It also takes more time and the retrieved contents are not exactly always same. novel internet image search require user only click on one image as query and images from the remaining images retrieved by text based search are re-ranked based on visual and texture feature. In Novel image re-ranking framework, offline learns different visual semantic spaces for different query keywords through keyword expansions. At the online stage, images are re-ranked by comparing their semantic signatures obtained from the visual semantic space specified by the query keyword.
\end{abstract}

Keywords: Image Search, Image Re-ranking, Image Retrieval, Semantic Signatures

\section{Introduction}

"Image Retrieval" is the process of finding the relevant images based on the user specified query keywords from the large image database. Nowadays, image collection scheme in web is growing dynamically. The aim of the image search is to retrieve the relevant image with respect to user query from a large image database. Image re-ranking improves the results of web based image search. Image retrieval is a key issue of user concern. An image retrieval system is a computer system for browsing, searching and retrieving images from a large database. Normal way of image retrieval is the text based image retrieval technique. Text based image retrieval needs rich semantic textual description of web images .This technique is popular but needs very specific description of the query which is tedious and not always possible.

Image re-ranking, as an effective way to improve the results of web-based image search, has been adopted by current commercial search engines. Given a query keyword, pools of images are first retrieved by the search engine based on textual information. By asking the user to select a query Image from the pool, the remaining images are re-ranked based on their visual similarities with the query image. Most of the web search engine is use keywords as queries. In our day today life billions of images are uploaded on web. It is challenging process to retrieve the images on web. Accurately describe the visual content, its very difficult to user because user only use keyword and hence text-based image search suffers from the ambiguity of query keywords. System user use query keyword and he expect to retrieve certain type of images. Search engine for example, using "apple" as query, the retrieved images belong to different categories, such as "red apple", "apple logo", and "apple laptop".

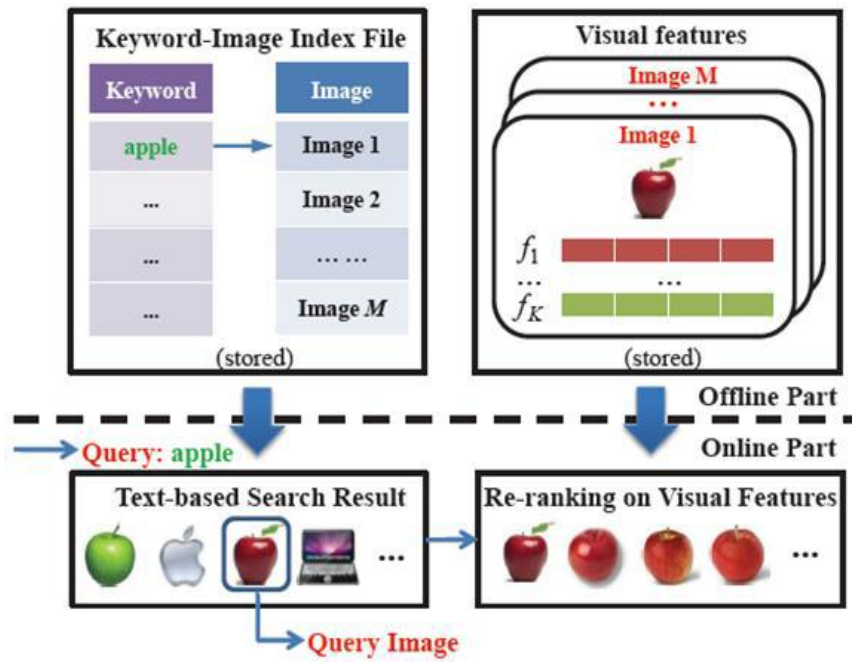

Figure 1: Image re-ranking Framework [1]

As shown in fig. Query keyword= "apple" which is input by user, according to a stored word-image index file, a pool of images relevant to the query keyword are retrieved by the search engine. By asking a user to select a query image, which reflects the user's search intention, from the pool, the remaining images in the pool are re-ranked based on their visual similarities with the query image.

The visual features of images are pre-computed offline and stored by the search engine. The main online computational cost of image re-ranking is on comparing visual features. In order to achieve high efficiency, the visual feature vectors need to be short and their matching needs to be fast.

\section{Literature Survey}

For TBIR (Text based image retrieval) requires a rich semantic textual description of web images. In this image are retrieved by using text keyword. TBIR are very famous, but it requires meaningful and specific description of the query, 


\section{International Journal of Science and Research (IJSR) \\ ISSN (Online): 2319-7064}

Index Copernicus Value (2013): 6.14 | Impact Factor (2015): 6.391

which is not always possible. The disadvantage of TBIR is in a huge collection of image it is not feasible and using only the query keyword image cannot be described properly as user need. It overcome in CBIR. Content-based search analyzes the context of the image instead of the meta data like description, keyword or tags of the image.[2]. Yuxinchen et al[3] Proposed vertical image search engine. It's complete both visual and textual feature of the image which improves performance of image retrieval. iLike system uses the parser to find a sample or pattern and text description of the link and the image. The advantage of this system is bridging the semantic gap and disadvantage is its only use text and result are not filtered.

Cui et al.[4] Proposed image re-ranking approach. In which limited user's effort to just one- click feedback. It is very simple image re-ranking approach has been adopted by Popular web scale engine like Google, bing as find Similar images function. Hybrid method [5] which used combined approach. Used both textual and visual feature and measure link based and content based similarity. Google Similarity [6] evaluates the similarity between word and phrase based on information distance. Here WWW uses as database and Google use as a search engine. Lei wu [7] proposed Flicker Distance. Flicker distance depends on visual information. Flicker distance measures the visual correlation between concepts. The Flicker website used for a collection of images. Visual information measures the proper relationship of concept. (Homogeneous) Image rich information retrieval [8] overcomes problem of information retrieval. MOKSimRank improves speed Sim-Rank. It extends into HMoK-SimRank which used to compute link based similarity in weighted homogeneous information network.

\section{Proposed System}

Here, Proposed System is Novel Image re-ranking framework [1]. It learns different visual semantic spaces for different query keywords individually and automatically. Ex. if the query keyword is "apple", the semantic concepts of "mountains" and "Paris" are unlikely to be relevant and can be ignored. Instead, the semantic concepts of "computers" and "fruit" will be used to learn the visual semantic space related to "apple".

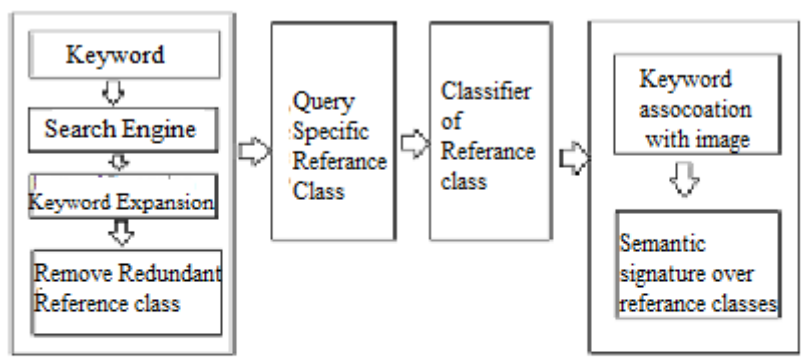

Offline Part

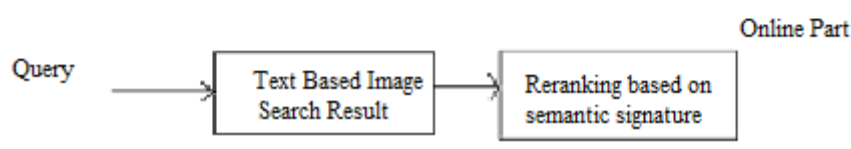

Figure 2: System Architecture
There are 2 parts offline and online parts. At offline stage Image re-ranking framework automatically learns different Semantic spaces for different query keywords. Semantic signature of query keyword from user is calculated and stored in the database. Reference classes representing different concepts related to query keywords are automatically discovered. As shown in Figure 3, for a query keyword (e.g. apple), a set of most relevant keyword expansions (such as red apple and apple macbook) are automatically selected utilizing both textual and visual information. Set of keyword expansions define reference classes for different keywords. A multi class classifier is trained on training set of reference classes. If there are $\mathrm{k}$ types of visual and textual features like color, shape, texture we can combine them to train single classifier.

At online stage pool of images are retrieved according to query keyword. Once user chooses query image semantic signatures are used to compute similarities of image with pre-computed semantic signatures.

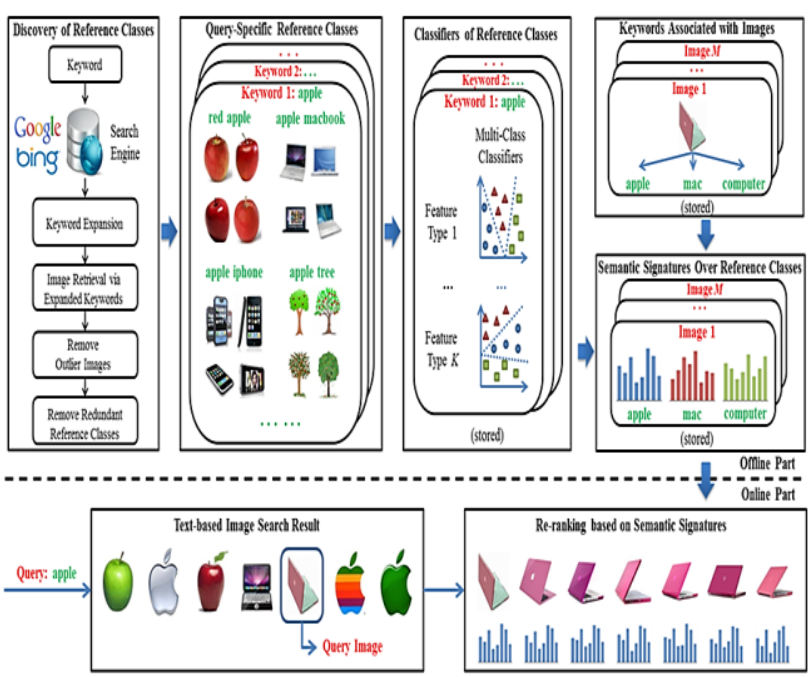

Figure 3: New Image Reranking Framework

The conventional framework compares images based upon their visual features. The length of these visual features is much longer than that of the semantic signatures used in the new framework. Hence, the computational cost is higher. Compared with the conventional image re-ranking diagram in Figure. 3, the new approach is much more efficient at the online stage, because the main online computational cost is on comparing semantic signatures and the lengths of semantic signatures are much shorter than those of low-level visual features. The advantages of this system is which improve efficiency and Visual features of thousands of dimensions can be projected to the semantic signatures as short as 25 dimensions. Disadvantage is it does not remove duplicate image.

Next survey on Sim-Rank algorithm, which compute the link based similarity. It is based on "Two nodes are similar"if they link by similar node in network. Among algorithms that compute object similarity in information networks, SimRank is one of the most popular, but it is very expensive to calculate and the similarity is only based on the link information. MoK-SimRank to significantly improve the 
speed of SimRank, and introduce its extension HMokSimRank[8] to work on weighted heterogeneous information networks. Heterogeneous minimum order k-SimRank (HMok-SimRank) to compute link-based similarity in weighted heterogeneous information networks. Integrated learning similarity algorithm IWSL[8] to provide a novel way of integrating both link and content information. IWSL performs content and link reinforcement style learning with either global or local feature weight learning.

\section{Conclusion}

Hence we conclude that ,In online based search we can efficiently find Object similarity using Sim-Rank algorithm. In weighted hetero-generous image-rich information networks, HMok-SimRank algorithm efficiently finds weighted link-based similarity. This method is more popular and faster. It also retrieve relevant image as per users query. The IWSL (Integrated Weighted Similarity Learning) algorithm compute both link based and content based similarity in weighted heterogeneous information network.

\section{References}

[1] X. wang, S.Qiu, K.Liu and X.tang, Web Image ReRanking Using Query-Specific Semantic Signatures. IEEE Trans. Pattern Pattern Analysis and Machine Intelligence, vol. 25, no. 10,pp. 1342-1353, July 2014.

[2] Y. Rui, T. S. Huang, M. Ortega, and S. Mehrotra. Relevance feedback: a power tool for interactive content-based image retrieval. IEEE Trans. on Circuits and Systems for Video Technology, 1998.

[3] Y.chen, H.Sampauoth kumar,B.luo, iLike:Bridging the Semantic Gap in vertical Image Search by Integrating Text and Visual Features. IEEE Trans.Pattern Analysis and Machine Intelligence, vol. 34, no. 7,pp. 1342-1353, July 2013

[4] J. Cui, F. Wen, and X. Tang. Intentsearch: Interactive on-line image search re-ranking. In Proc. ACM Multimedia. ACM, 2008.

[5] V.Abinaya1, Mr.A.R.Ashok Kumar2, Prof.D.Durai Kumar3, IJREAT, A Hybrid Similarity Measures For Integration In Image-Rich Information Networks.

[6] Cilibrasi, R.L. and P.M.B. Vitanyi, The Google SimilarityDistance, IEEE Trans.Knowledge and Data Eng.2007

[7] L. Wu, X.-S. Hua, N. Yu, W.-Y. Ma, and S. Li, Flickr Distance, Proc. 16th ACM IntL conf. Multimedia, pp. 31-40, 2008.

[8] Xin Jin, Jiebo Luo, Fellow, IEEE, Jie Yu,Reinforced Similarity Integration In Image Rich Information Networks feb.2013.

\section{Author Profile}

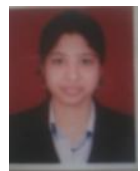

Vandana R Nangare Student at Zeal Education Societys, Zeal college of Engineering and Research, Department of Computer engineering, pune411046,India. 